\title{
A Stochastic Inventory System with Postponed Demands and Infinite Pool in Discrete-Time Setup
}

\author{
Velusamy Radhamani • P. Chitra Devi • \\ Balasubramanian Sivakumar
}

Received: 23 May 2014/Revised: 11 November 2014/ Accepted: 16 November 2014/

Published online: 23 December 2014

(C) Operations Research Society of China, Periodicals Agency of Shanghai University, and SpringerVerlag Berlin Heidelberg 2014

\begin{abstract}
In this article, we consider a discrete-time inventory model in which demands arrive according to a discrete Markovian arrival process. The inventory is replenished according to an $(s, S)$ policy, and the lead time is assumed to follow a discrete phase-type distribution. The demands that occur during stock-out periods either enter a pool which has an infinite capacity or leave the system with a predefined probability. The demands in the pool are selected one by one, if the on-hand inventory level is above $s+1$, and the interval time between any two successive selections is assumed to have a discrete phase-type distribution. The joint probability distribution of the number of customers in the pool and the inventory level is obtained in the steady-state case. We derive the system performance measures under steady state and using these measures, the total expected cost rate of the system is calculated. The impacts of arrival rate on the performance measures are graphically illustrated. Finally, we study the impact of cost on the optimal values of the total expected cost rate, inventory level and the reorder point.
\end{abstract}

Keywords $(s, S)$ Policy · Discrete-time inventory system · Discrete Markovian arrival process $\cdot$ Discrete phase-type distribution $\cdot$ Postponed demands

The research was supported by Council of Scientific and Industrial Research, India, research award (No. 25(0183)/10/EMR-II).

\footnotetext{
V. Radhamani · B. Sivakumar $(\bowtie)$

Department of Applied Mathematics and Statistics, Madurai Kamaraj Univesity, Madurai 625021, Tamil Nadu, India

e-mail: sivabkumar@yahoo.com

P. C. Devi

Department of Mathematics, G.T.N. Arts College, Dindigul 624005, Tamil Nadu, India
} 


\section{Introduction}

Continuous review inventory system with postponed demands has received considerable attention in the last few decades. [12] considered an inventory system with Poisson demand, exponential lead time and assumed that the pooled customers are selected according to an exponentially distributed time. [23] considered an inventory model in which the demands occur according to a Markovian arrival process, lead time is distributed as phase type, life time for the items in the stock has exponential distribution, and the pooled customers are selected exponentially. [18] dealt an inventory system in which the positive and negative demands occur according to independent Markovian arrival processes and the lead time of the reorder, the life time of the items, the inter-selection time of customers from the pool and the reneging time points of the customers in the pool have independent exponential distributions. [24] considered an inventory system with independent Markovian arrival processes for both positive and negative demands, exponential distribution for the lead time, exponential life times for each item in the stock and an infinite pool size.

In all the above models, the authors assumed that all the system events are monitored continuously and that the time axis is continuous. Although many inventory systems are conveniently characterized by fixed-length intervals during which events occur and decisions are made, only few articles in the literature dealt with discrete-time inventory models. However, there is a growing research interest in discrete-time queues (see $[2-4,26]$ ), mainly motivated by their applications in computer and communication systems where the time axis is often slotted.

The first paper on discrete-time inventory models was [7]. This paper analysed a Markovian inventory model for perishable commodities, in which the arrivals of items into the system as well as the demands for these items were assumed to be discrete random variables having common supports $0,1, \cdots$. The items are assumed to have the life span of $N$ item units. Some characteristics of this model were derived for the case of two and three age categories.

Lian and Liu [14] developed a discrete-time inventory model with geometric inter-demand times and constant life time. They assumed that the demands arrive in batches and that the batch size was random. They also assumed that the lead time was zero and full backlogging of demands. They used matrix-analytic methods to construct a discrete-time Markov chain at the inventory level, and they obtained a closed-form average cost function.

Abboud [1] analysed a discrete-time Markov model for production inventory systems with machine breakdowns. He assumed that the demand and production rates were constant, and the production rate was greater than the demand rate. The failure times and the repair times were independently distributed as geometric and the demands that occur during stock out were back-ordered.

Lian et al. [15] discussed a discrete-time model for common lifetime inventory systems. They assumed that the demand arrives in batches according to a discrete phase-type renewal process, and the lifetime of an item had a discrete $\mathrm{PH}$ distribution. They assumed that the supply of the order was instantaneous, and unmet demands are back-ordered. 
In this work, we use the concepts of discrete phase-type distribution and discrete Markovian arrival process (DMAP), and hence we give an introduction and notation on these concepts. The MAP was a class of the Markov counting process introduced by [20] as a generalization of the Poisson process which was well suited for matrixanalytic method and numerical investigations. The research reported by $[16,17]$ suggested a convenient notation which was better suited for a general discussion than the one which was originally used. A highly accessible discussion of the MAP, with many examples, may be found in [16]. A partly expository paper discussing how the MAP can be used qualitatively to model point processes with certain "bursty" feature given by [22]. For more details on the DMAP, we refer to readers [9] and [11]. The following is a brief informal description of the DMAP, which should be adequate for this paper. Let $D$ be an irreducible stochastic matrix of order $n$ and let $D_{0}$ and $D_{1}$ be two sub-stochastic matrices whose sum is $D$ such that the matrix $I-D_{0}$ is non-singular. The element $\left[D_{0}\right]_{i j}$ represents a transition from phase $i$ to phase $j$ which is associated with a non-occurrence of an event (such as arrival), and the element $\left[D_{1}\right]_{i j}$ represents a transition from phase $i$ to phase $j$ which is associated with an occurrence of the above event. Let $\eta D=\eta$ with $\eta e=1$. Then the rate of occurrence of the event is $\lambda=\eta D_{1} e$, where $\eta$ is the stationary probability distribution of the transition probability matrix $D$. The sequence of time points of these transitions forms a stochastic process which is known as DMAP with parameters $n, D_{0}$ and $D_{1}$. We represent such a DMAP by $\left(D_{0}, D_{1}\right)_{n}$.

The discrete phase-type distribution was introduced in the mid 1970s, see [19]. However, more researchers have been focusing on the studies of the continuous phase-type distributions. Detailed discussions of continuous phase-type distributions can be found in [21] and [13]. Brief overviews of either discrete or continuous phase-type distributions and their properties can be found in $[5,6,8,10]$ and the references therein.

We briefly describe the phase-type distribution. Consider a Markov chain with $m$ transient states and one absorbing state, say $\mathbf{0}$. It has an associated transition probability matrix

$$
\tilde{T}=\left[\begin{array}{cc}
1 & \mathbf{0} \\
T^{0} & T
\end{array}\right],
$$

where $T^{0}=\left(t_{10}, t_{20}, \cdots, t_{m 0}\right)^{\prime}$, and $T=\left(t_{i j}\right)_{m \times m}$. The matrix $T$ is a sub-stochastic matrix, holding the transition probabilities among the $m$ transient states, and $T^{0}$ contains the absorption probabilities into state 0 from the transient states. Clearly, $T^{0}$ satisfies $T e+T^{0}=e$, where $e$ is a column vector of appropriate dimension containing all ones. The initial state of this Markov chain is chosen according to the probability vector $\left(b_{0}, \beta\right)$ where $b_{0} \geqslant 0$ and $\beta$ is a row vector of size $m$. Once the initial state of the Markov chain is fixed, the chain gets absorbed after visiting various states. The states are also called phases, and the time till absorption is a sum of exponentially distributed random variables with parameters depending on the phases visited. Hence, the distribution of time till absorption is called phase-type distribution. The mean of the phase-type distribution is given by $\mu=\beta(I-T)^{-1} e$. 
This phase-type distribution is represented by $(\beta, T)_{m}$. If, at every time of absorption, the Markov chain is started by selecting the initial state according to the probability vector $\left(b_{0}, \beta\right), b_{0} \geqslant 0$ (usually referred to as initialization), then the point process of times at which the chain is absorbed (or initialized) forms a phasetype renewal process.

Recently, [25] analysed a discrete-time inventory system with postponed demand, and the pool has finite capacity. They considered the demands arrived according to discrete-time Markovian arrival process, and the lead time and pool customer's selection follow discrete phase-type distribution.

In this paper, we extend the work of [25] by assuming that the capacity of the pool is infinite. This mathematical model has a real-life application in many industries, including the garments industry, the retailer's shop, etc., to satisfy the demand of customer. For example, in a retailer shop, during the stock-out period, to avoid the demand lost, an arriving customer is offered a choice of postponement by the retailer. If this customer accepts the postponement, then the retailer will satisfy this postponed customer after the replenishment of items.

The rest of the paper is organized as follows. In Sect. 2, we describe the mathematical model for the problem under consideration. The stability analysis and steady-state analysis of the model are presented in Sect. 3, and some important system performance measures and the total expected cost rates are derived in Sect. 4. In Sect. 5, we provide numerical illustrations of the results.

\section{Notation}

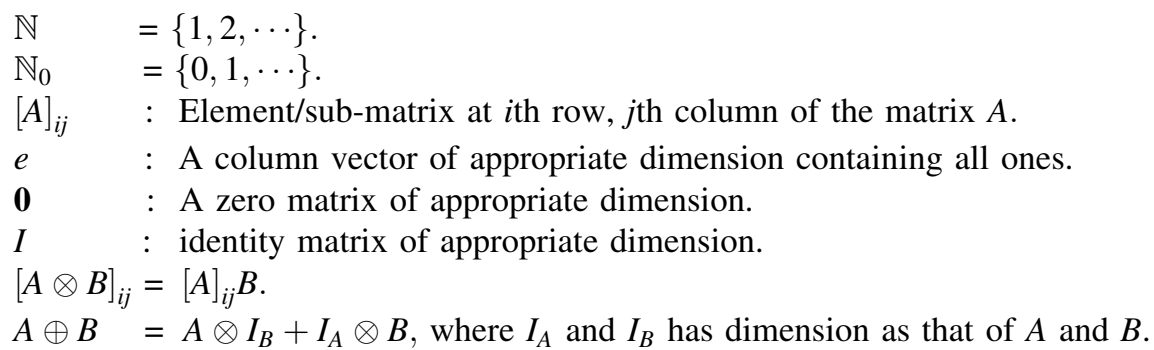

\section{Mathematical Model}

Consider a discrete-time inventory system in which all system-related activities occur at discrete-time points only. We describe the discrete-time system as defined by [9]. The system is monitored at time epochs sequentially numbered $0,1, \cdots$, and all events which occur between epoch $t$ and $t+1$ are assumed to occur at epoch $t+1$.

Now we describe the mathematical model of the system considered in this work as follows: 
- The inventory order strategy is $(s, S)$ policy.

- The unit demands to occur according to a DMAP with representation $\left(D_{0}, D_{1}\right)_{n}$.

- The lead time for the supply of the reorder is assumed to have a discrete phasetype distribution with representation $\left(\alpha_{1}, T_{1}\right)_{m_{1}}$.

- Any arriving demand, when the inventory level is zero, is offered a choice of either leaving the system immediately or being postponed until the ordered items are received. We assume that the demanding customer accepts the offer of postponement according to an independent Bernoulli trial with probability $p(0 \leqslant p<1)$ and with probability $q(=1-p)$ and the customer declines the offer and leaves the system.

- The customers who opted for postponement of their demands are retained in a pool, which has an infinite capacity.

- Only if the inventory level is greater than $s+1$, the customers in the pool can be selected one by one according to FCFS discipline.

- The time between two successive selections is assumed to have a discrete phasetype distribution with representation $\left(\alpha_{2}, T_{2}\right)_{m_{2}}$, and the rate of selection of demands from the pool is given by $\alpha=\left[\alpha_{2}\left(I-T_{2}\right)^{-1} e\right]^{-1}$.

\section{Analysis}

We have the following random variables defined at time $t$.

$L_{t}:$ the inventory level,

$X_{t}$ : the number of demands in the pool,

$J_{t}^{D}:$ the phase of the MAP associated with demand process,

$J_{t}^{L}$ : the phase of the distribution of lead time of an order, if order is placed already,

$J_{t}^{S}$ : the phase of the distribution associated with the selection of demand from pool.

It may be noted that $J_{t}^{L}$ and $J_{t}^{S}$ do not arise when the inventory level $L_{t}>s$ and the number of customers in the pool $X_{t}=0$ and that $J_{t}^{L}$ does not arise when $L_{t}>s$ and $X_{t}>0$. In the same way, $J_{t}^{S}$ does not arise if $L_{t} \leqslant s+1$. For clarity, we shall assume and write 0 value for the state of random variables, which do not arise in the above cases. It may be noted that for these random variables, the associated Markov chain has 0 as the absorbing state and hence when these random variables do not arise, one can assume that the associated Markov chain is in absorbing state 0 .

When multiple events can occur in a time slot, for mathematical tractability, we assume the following order: (1) the materialization of an order, (2) the satisfaction of a demand and (3) the selection of demand from the pool.

In the case of random variables with phase-type distribution, the initial state has to be fixed by the associated initial probability vector. For more than one such initialization, we assume the following order of initialization: (i) selection process of customers from the pool and (ii) lead time of an order. 
Table 1 State space of the model

\begin{tabular}{|c|c|c|c|c|c|}
\hline \multicolumn{6}{|c|}{ States of variables } \\
\hline Block & $X_{t}$ & $L_{t}$ & $J_{t}^{D}$ & $J_{t}^{L}$ & $J_{t}^{S}$ \\
\hline 0 & $x=0,1, \cdots$ & $l=0,1, \cdots, s$ & $j=1,2, \cdots, n$ & $j_{1}=1,2, \cdots, m_{1}$ & - \\
\hline 1 & $x=0$ & $l=s+1, s+2, \cdots, s$ & $j=1,2, \cdots, n$ & - & - \\
\hline 2 & $x=1,2, \cdots$ & $l=s+1$ & $j=1,2, \cdots, n$ & - & - \\
\hline 3 & $x=1,2, \cdots$ & $l=s+2, \cdots, S$ & $j=1,2, \cdots, n$ & - & $j_{2}=1,2, \cdots, m_{2}$ \\
\hline
\end{tabular}

It may be noted that if the replenishment, the occurrence of a demand and the selection from the pool occur in a time slot, then the inventory level becomes at least $Q-2$. In order to place a subsequent order, this level must be greater than the reorder level $s$. Otherwise, there is a positive probability that the stock may face a perpetual shortage. Hence, we assume $Q \geqslant s+2$.

The possible states of the stochastic process $\left\{\left(X_{t}, L_{t}, J_{t}^{D}, J_{t}^{L}, J_{t}^{S}\right), t \in \mathbb{N}_{0}\right\}$ are given in Table 1. In Table 1, the blocks represent the different set of states for 5tuples $\left(x, l, j, j_{1}, j_{2}\right)$, and the union of these four blocks gives the state space $E$ of the process $\left\{\left(X_{t}, L_{t}, J_{t}^{D}, J_{t}^{L}, J_{t}^{S}\right), t \in \mathbb{N}_{0}\right\}$.

Now we check the Markov property of the stochastic process $\left\{\left(X_{t}, L_{t}, J_{t}^{D}, J_{t}^{L}, J_{t}^{S}\right) ; t \in \mathbb{N}_{0}\right\}$. i.e.

$$
\begin{aligned}
& \operatorname{Pr}\left(\left(X_{n+1}=x_{n+1}, L_{n+1}=l_{n+1}, J_{n+1}^{D}=j_{n+1}, J_{n+1}^{L}=j_{1_{n+1}}, J_{n+1}^{S}=j_{2_{n+1}}\right)\right. \\
& \quad \mid\left(X_{n}=x_{n}, L_{n}=l_{n}, J_{n}^{D}=j_{n}, J_{n}^{L}=j_{1_{n}}, J_{n}^{S}=j_{2_{n}}\right),\left(X_{n-1}, L_{n-1}, J_{n-1}^{D}, J_{n-1}^{L}, J_{n-1}^{S}\right), \cdots \\
& \left.\quad\left(X_{0}, L_{0}, J_{0}^{D}, J_{0}^{L}, J_{0}^{S}\right)\right)
\end{aligned}
$$

depends only on $\left(x_{n}, l_{n}, j_{n}, j_{1_{n}}, j_{2_{n}}\right)$ and $\left(x_{n+1}, l_{n+1}, j_{n+1}, j_{1_{n+1}}, j_{2_{n+1}}\right)$. For example, consider the case $\left(X_{n}=x_{n}, L_{n}=0, J_{n}^{D}=j_{n}, J_{n}^{L}=j_{1_{n}}\right) \quad$ and $\left(X_{n+1}=x_{n}+1, L_{n+1}=0, J_{n+1}=j_{n+1}, J_{n+1}^{L}=j_{1_{n+1}}\right)$. In order to move to the state $\left(x_{n+1}, 0, j_{n+1}, j_{1_{n+1}}\right)$ at time $n+1$,

i. An arrival with phase changes from $j_{n}$ to $j_{n+1}\left(j_{n}, j_{n+1}=1,2, \cdots, n\right)$ must occur and this arrived customer must accept the postponement.

ii. Also no replenishment takes place, but the phase must change from $j_{1_{n}}$ to $j_{1_{n+1}}\left(j_{1_{n}}, j_{1_{n+1}}=1,2, \cdots, m_{1}\right)$.

The probability for the first event is $p\left[D_{1}\right]_{j_{n} j_{n+1}}$ and for the second event is $\left[T_{1}\right]_{j_{1 n} j_{1 n+1}}$. Therefore, the value of Eq. 3.1 is $\left[p\left[D_{1}\right]_{j_{n} j_{n+1}}\left[T_{1}\right]_{j_{1_{n} j_{1 n+1}}}\right.$. Since the probability depends only on $\left[\left(x_{n+1}, 0, j_{n+1}, j_{1_{n+1}}\right)\right.$ and $\left[\left(x_{n}+1,0, j_{n}, j_{1_{n}}\right)\right.$, the stochastic process satisfies the Markov property. Similarly, we can write all the transition probability values. Therefore, $\left[\left\{\left(X_{t}, L_{t}, J_{t}^{D}, J_{t}^{L}, J_{t}^{S}\right) ; t \in \mathbb{N}_{0}\right\}\right.$ is a discrete-time Markov chain with the state space $E$. 
Table 2 Description of the states

\begin{tabular}{|c|c|c|c|}
\hline Class & From & To & Due to \\
\hline \multirow[t]{13}{*}{$\langle i\rangle$ to $\langle i\rangle$} & $\begin{array}{l}\langle i, 0\rangle \\
i=0,1, \cdots\end{array}$ & $\langle i, 0\rangle$ & $\begin{array}{l}\text { No demand \& no replenishment, } \\
\text { demand not joining pool } \\
\& \text { no replenishment }\end{array}$ \\
\hline & $\langle i, l\rangle$ & $\langle i, l\rangle$ & No demand \& no replenishment \\
\hline & $i=0,1, \cdots, l=1,2, \cdots, s$ & $\langle i, l-1\rangle$ & Demand \& no replenishment \\
\hline & $\langle i, s+1\rangle$ & $\langle i, s+1\rangle$ & No demand \\
\hline & $i=1,2, \cdots$ & $\langle i, s\rangle$ & Demand \\
\hline & $\langle 0, l\rangle$ & $\langle 0, l\rangle$ & No demand \\
\hline & $l=s+2, \cdots, S$ & $\langle 0, l-1\rangle$ & Demand \\
\hline & $\langle i, s+2\rangle$ & $\langle i, s+2\rangle$ & No demand \& no selection from pool \\
\hline & $i=1,2, \cdots$ & $\langle i, s+1\rangle$ & Demand \\
\hline & $\langle i, l\rangle$ & $\langle i, l\rangle$ & No Demand \& No Selection from Pool \\
\hline & $i=1,2, \cdots, l=s+3, \cdots, S$ & $\langle i, l-1\rangle$ & Demand \& No Selection from Pool \\
\hline & $\langle i, l\rangle$ & $\langle i, l+Q\rangle$ & No demand \& replenishment \\
\hline & $i=1,2, \cdots, l=0,1, \cdots, s$ & $\langle i, l+Q-1\rangle$ & Demand \& replenishment \\
\hline \multirow[t]{2}{*}{$\langle i\rangle$ to $\langle i+1\rangle$} & $\langle i, 0\rangle$ & $\langle i+1,0\rangle$ & Demand \& no replenishment \\
\hline & $i=0,1, \cdots$ & & \\
\hline \multirow[t]{2}{*}{$\langle i\rangle$ to $\langle i-1\rangle$} & $\langle i, l\rangle$ & $\langle i-1, l-1\rangle$ & No demand \& selection from pool \\
\hline & $i=0,1, \cdots, l=s+2, \cdots, S$ & $\langle i-1, l-2\rangle$ & Demand \& selection from pool \\
\hline
\end{tabular}

* Here, we specifically need $Q-1 \geqslant s+2 \Rightarrow Q \geqslant s+3 \Rightarrow Q>s+2$

The possible transitions of states in the discrete-time Markov chain are listed in Table 2.

The transitions other than those listed in the Table 2 cannot occur.

The transition probability matrix $P$ of the discrete-time Markov chain $\left\{\left(X_{t}, L_{t}, J_{t}^{D}, J_{t}^{L}, J_{t}^{S}\right) ; t \in \mathbb{N}_{0}\right\}$ is a Quasi-Birth-and-Death process. Because of the assumptions made in this model, the outer random variable, i.e. the number of demands in the pool, $X_{t}$

i. Increases by one i.e. a birth occurs when the demands arrive during a stockout period with acceptance of postponement, and no replenishment takes place at that time epoch.

ii. Decreases by one i.e. a death occurs due to pool customer selection when the inventory level is above $s+1$ with or without demand occur.

iii. All other events occur in the same level.

According to the possible transitions given in Table 2, the transitions from $\langle 0\rangle$ to $\langle 1\rangle,\langle 0\rangle$ to $\langle 0\rangle$ and $\langle 1\rangle$ to $\langle 0\rangle$ are considered as block matrix $B_{0}, B_{1}$ and $B_{2}$. The transitions $\langle i\rangle$ to $\langle i\rangle$ and $\langle i\rangle$ to $\langle i+1\rangle(i=1,2, \cdots)$ are considered as block matrix $A_{1}, A_{0}$ and $\langle i\rangle$ to $\langle i-1\rangle(i=2,3, \cdots)$ is considered as block matrix $A_{2}$. Then the transition probability matrix $P$ of the discrete-time Markov chain is given below: 


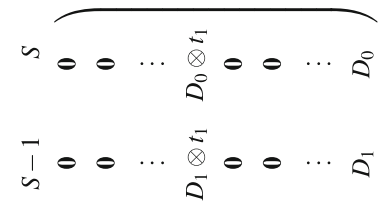

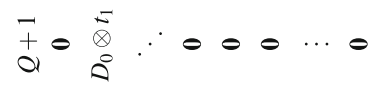

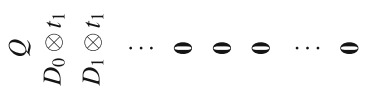$$
\overrightarrow{1}_{\vec{\sigma}}^{\vec{\sigma}} \theta \cdots \theta \theta \theta \cdots
$$

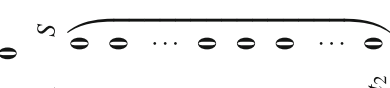

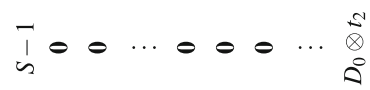

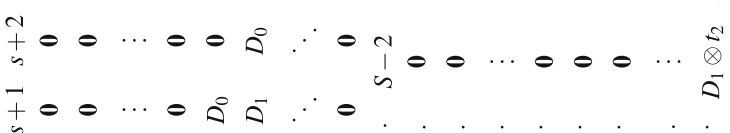

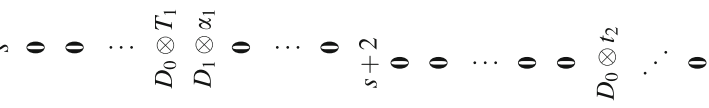

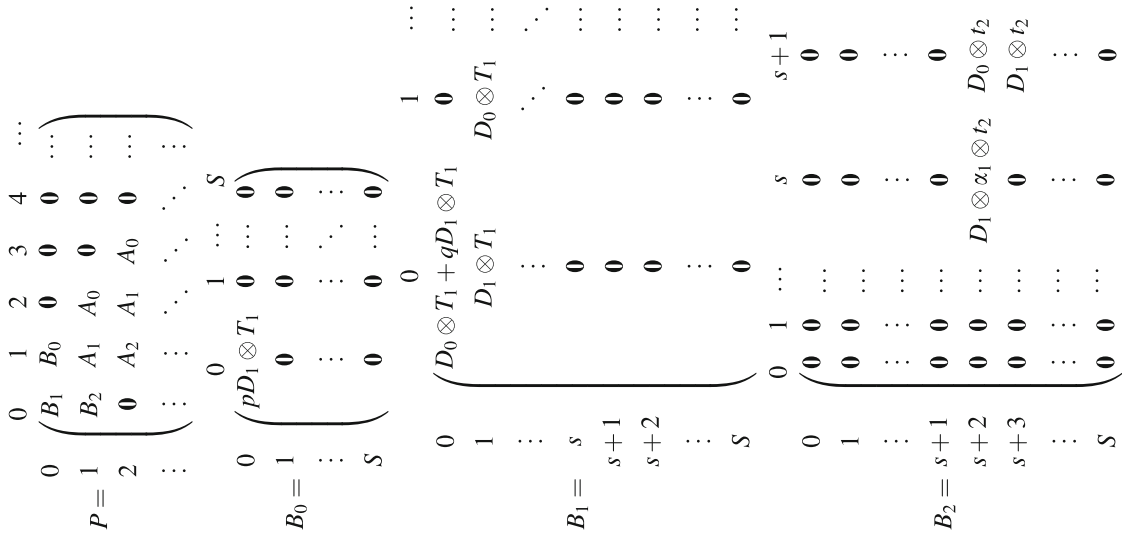



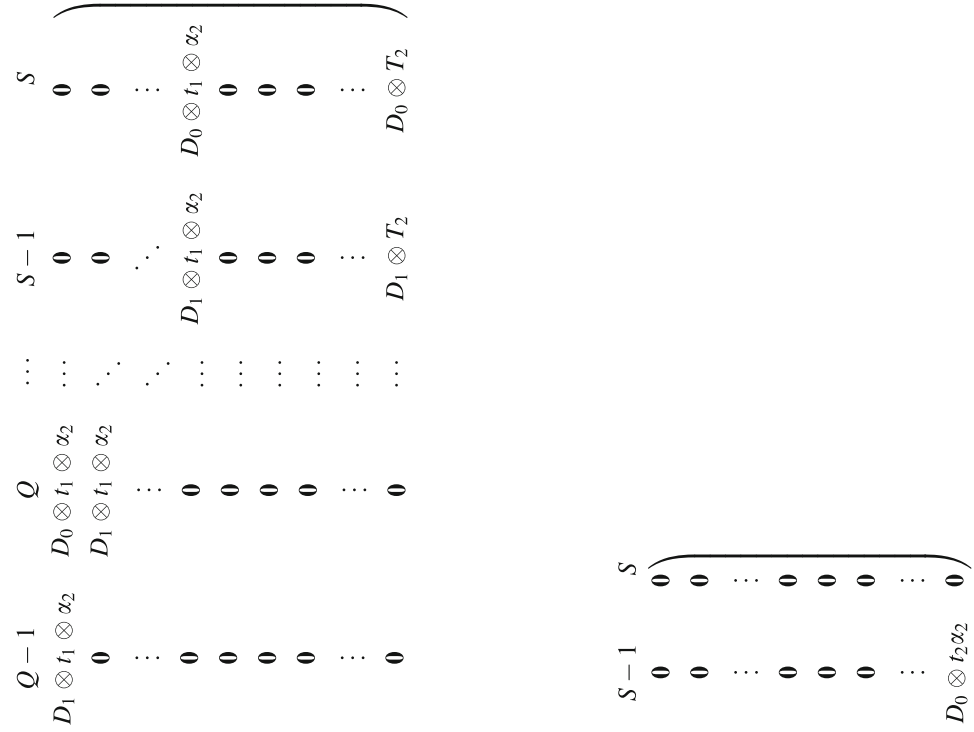

$+i \frac{0}{0} \frac{0}{4} \cdot \cdot 0$

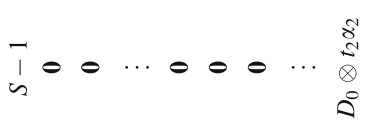

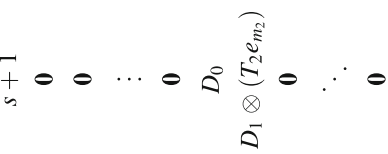

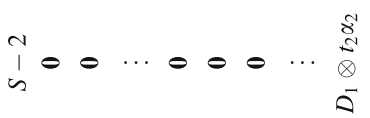

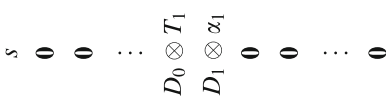

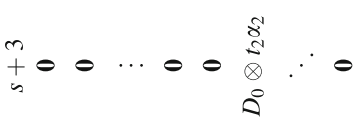

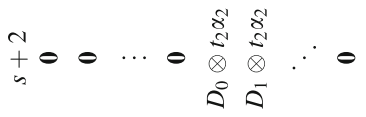

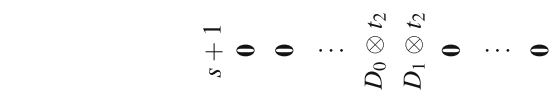

$-0 \underset{0}{R} . .0000$

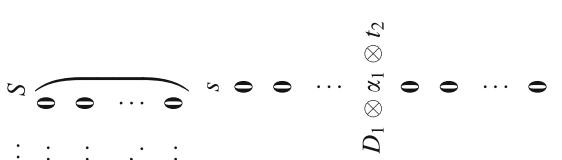

$\otimes$

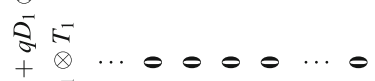
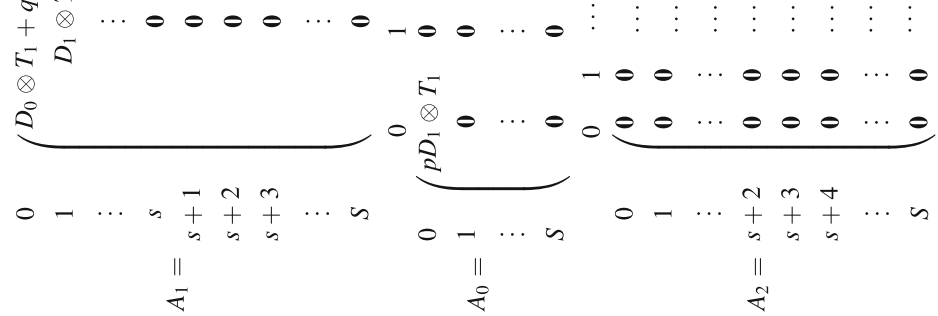
It may be noted that $B_{0}, B_{1}$ and $B_{2}$ are matrices of size $(s+1) n m_{1}+$ $Q n \times(s+1) n m_{1}+n+(Q-1) n m_{2}, \quad(s+1) n m_{1}+Q n \times(s+1) n m_{1}+Q n, \quad(s+$ 1) $n m_{1}+n+(Q-1) n m_{2} \times(s+1) n m_{1}+Q n$ and the size of the matrices $A_{0}, A_{1}$ and $A_{2}$ are $(s+1) n m_{1}+n+(Q-1) n m_{2} \times(s+1) n m_{1}+n+(Q-1) n m_{2}$.

\subsection{Stability Analysis}

In this section, we derive the necessary condition for the positive recurrence of the Markov chain $\left\{\left(X_{t}, L_{t}, J_{t}^{D}, J_{t}^{L}, J_{t}^{S}\right) ; t \in \mathbb{N}_{0}\right\}$. We first consider the stochastic matrix $A=A_{0}+A_{1}+A_{2}$, which is given by 

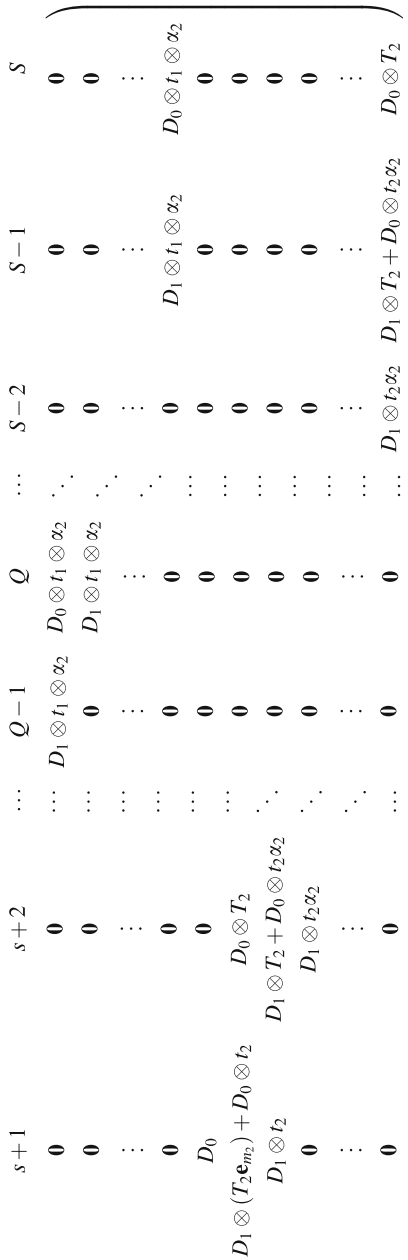

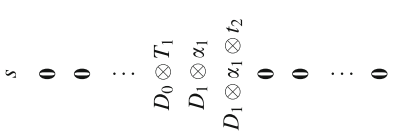
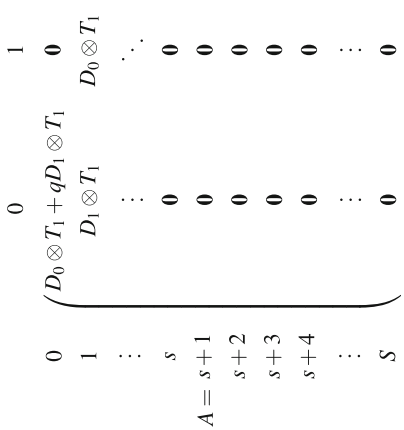
It can be seen from the structure of the transition probability matrix $A$ that the underlying Markov chain is irreducible and aperiodic. Since the state space of the underlying Markov chain is finite, it is also positive recurrent. Hence, the limiting distribution exists and it is independent of the initial state. Let

$$
\underline{\pi}=\left(\pi^{(0)}, \pi^{(1)}, \cdots, \pi^{(S)}\right)
$$

be the steady-state probability vector of $A$. Then, $\underline{\pi}$ can be shown to satisfy the following equations:

$$
\begin{aligned}
\underline{\pi} A & =\underline{\pi} \\
\text { and } \underline{\pi} e & =1 .
\end{aligned}
$$

In the next lemma, we give the condition for the positive recurrence of Markov chain $\left\{\left(X_{t}, L_{t}, J_{t}^{D}, J_{t}^{L}, J_{t}^{S}\right) ; t \in \mathbb{N}_{0}\right\}$.

Lemma 3.1 The discrete-time Markov chain $\left\{\left(X_{t}, L_{t}, J_{t}^{D}, J_{t}^{L}, J_{t}^{S}\right) ; t \in \mathbb{N}_{0}\right\}$ is positive recurrent if

$$
\begin{aligned}
\left(\pi^{(0)}\left(p D_{1} \otimes T_{1}\right)\right) e< & \left(\pi^{(s+2)}\left(D_{1} \otimes \alpha_{1} \otimes t_{2}\right)\right) e+\left(\pi^{(s+2)}\left(D_{0} \otimes t_{2}\right)\right) e+\left(\pi^{(s+3)}\left(D_{1} \otimes t_{2}\right)\right) e \\
& +\sum_{i=s+3}^{S}\left(\pi^{(i)}\left(D_{0} \otimes t_{2} \alpha_{2}\right)\right) e+\sum_{i=s+4}^{S}\left(\pi^{(i)}\left(D_{1} \otimes t_{2} \alpha_{2}\right)\right) e .
\end{aligned}
$$

Proof From the well-known result of [21] on the positive recurrence of $P$, we have

$$
\underline{\pi} A_{0} e<\underline{\pi} A_{2} e .
$$

By exploiting the structure of the matrices $A_{0}$ and $A_{2}$, and $\underline{\pi}$, the stated result follows.

\subsection{Steady-State Analysis}

It can be seen from the structure of the transition probability matrix $P$ and from the inequality (Eq. 3.2) that the discrete-time Markov chain $\left\{\left(X_{t}, L_{t}, J_{t}^{D}, J_{t}^{L}, J_{t}^{S}\right) ; t \in \mathbb{N}_{0}\right\}$ on $E$ is irreducible, aperiodic and positive recurrent.

Hence, the limiting distribution, defined by

$$
\phi^{\left(x, l, j, j_{1}, j_{2}\right)}=\lim _{n \rightarrow \infty} \operatorname{Pr}\left[X_{t}=x, L_{t}=l, J_{t}^{D}=j, J_{t}^{L}=j_{1}, J_{t}^{S}=j_{2} \mid X_{0}, L_{0}, J_{0}^{D}, J_{0}^{L}, J_{0}^{S}\right]
$$

exists and is independent of the initial state.

We group the probabilities $\phi^{\left(x, l, j, j_{1}, j_{2}\right)}$ as follows: 


$$
\begin{aligned}
\underline{\phi} & =\left(\underline{\phi}^{(0)}, \underline{\phi}^{(1)}, \cdots\right), \\
\underline{\phi}^{(x)} & =\left(\phi^{(x, 0)}, \phi^{(x, 1)}, \cdots, \phi^{(x, S)}\right), \quad x=0,1, \cdots, \\
\phi^{(x, l)} & =\left(\phi^{(x, l, 1)}, \phi^{(x, l, 2)}, \cdots, \phi^{(x, l, n)}\right), \quad x=0,1, \cdots, l=0,1, \cdots, S, \\
\phi^{(x, l, j)} & = \begin{cases}\left(\phi^{(x, l, j, 1)}, \phi^{(x, l, j, 2)}, \cdots, \phi^{\left(x, l, j, m_{1}\right)}\right), & x=0,1, \cdots, \quad l=0,1, \cdots, s, j=1,2, \cdots, n, \\
\left(\phi^{(x, l, j, 1)}, \phi^{(x, l, j, j)}, \cdots, \phi^{\left(x, l, j, m_{2}\right)}\right), \quad x=1,2, \cdots, l=s+2, s+3, \cdots, S, \quad j=1,2, \cdots, n .\end{cases}
\end{aligned}
$$

Then, the limiting probability distribution $\underline{\phi}$ satisfies the following equations:

$$
\underline{\phi P}=\underline{\phi} \quad \text { and } \quad \underline{\phi e}=1 .
$$

Theorem 3.2 When the stability condition (Eq. 3.2) holds good, the steady-state probability vector $\underline{\phi}$ is given by

$$
\underline{\phi}^{(x)}=\underline{\phi}^{(1)} \boldsymbol{R}^{x-1}, \quad x=2,3, \cdots,
$$

where the matrix $\boldsymbol{R}$ satisfies the quadratic equation

$$
\boldsymbol{R}^{2} A_{2}+\boldsymbol{R} A_{1}+A_{0}=\boldsymbol{R},
$$

and the vector $\underline{\phi}^{(0)}$ and $\underline{\phi}^{(1)}$ is obtained by solving

$$
\underline{\phi}^{(0)}\left(B_{1}-I\right)+\underline{\phi}^{(1)} B_{2}=0
$$

and

$$
\underline{\phi}^{(0)} B_{0}+\underline{\phi}^{(1)}\left(A_{1}+\boldsymbol{R} A_{2}-I\right)=0,
$$

subject to the normalizing condition,

$$
\underline{\phi}^{(0)} e+\underline{\phi}^{(1)}(I-\boldsymbol{R})^{-1} e=1 .
$$

Proof The theorem follows from the well-known result on matrix geometric methods ([21]).

\subsection{Computation of $\boldsymbol{R}$ Matrix}

In this subsection and the next one, we present an algorithmic procedure for computing the $\boldsymbol{R}$ matrix and the vector $\phi$, which are the main ingredients for discussing the qualitative behaviour of the model under study.

The square matrix $\boldsymbol{R}$ of dimension $(s+1) n m_{1}+n+(Q-1) n m_{2}$ can be computed as follows: We note that as $A_{0}$ has a non-zero entry in its first $n m_{1}$ rows only, and the matrix $\boldsymbol{R}$ also has non-zero entries in its first $n m_{1}$ rows only. Hence, we write 


$$
\boldsymbol{R}=\left(\begin{array}{cccccc}
\boldsymbol{R}_{0} & \boldsymbol{R}_{1} & \boldsymbol{R}_{2} & \cdots & \cdots & \boldsymbol{R}_{s} \\
0 & 0 & 0 & \cdots & \cdots & 0 \\
\vdots & \vdots & \vdots & \vdots & \vdots & \vdots \\
0 & 0 & 0 & \cdots & \cdots & 0
\end{array}\right)
$$

Due to this special form of $\boldsymbol{R}$, we note

$$
\boldsymbol{R}^{k}=\left(\begin{array}{cccccc}
\boldsymbol{R}_{0}^{k} & \boldsymbol{R}_{0}^{k-1} \boldsymbol{R}_{1} & \boldsymbol{R}_{0}^{k-1} \boldsymbol{R}_{2} & \cdots & \cdots & \boldsymbol{R}_{0}^{k-1} \boldsymbol{R}_{s} \\
0 & 0 & 0 & \cdots & \cdots & 0 \\
\vdots & \vdots & \vdots & \vdots & \vdots & \vdots \\
0 & 0 & 0 & \cdots & \cdots & 0
\end{array}\right), \quad k=1,2, \cdots
$$

This form is exploited in the computation of $\boldsymbol{R}$ using (Eq. 3.3). The relevant equations are given in Appendix 1.

\subsection{Computation of the Vectors $\phi^{(0)}$ and $\phi^{(1)}$}

Due to the special structure of $\boldsymbol{R}$ matrix as well as the coefficient matrices $B_{0}, B_{1}, B_{2}, A_{1}$ and $A_{2}$, the vectors $\underline{\phi}^{(0)}$ and $\underline{\phi}^{(1)}$ are computed from the Eqs. 3.4, 3.5 and 3.6. The detailed computation of this vector is given in Appendix 2.

\section{System Performance Measures}

In this section, we derive some system performance measures which are very useful in qualitative interpretation of the model under study.

1. Expected inventory level Let $\zeta_{I}$ denote the expected inventory level in the steady state. Since $\phi^{(x, l)}$ denotes the steady-state probability vector for $l$-th inventory with each component specifying a particular combination of the number of demands in the pool, that is the phase of the arrival process, the phase of the lead time process, if the order is placed, and the phase of the selection process, if the inventory level is above $s+1$, then $\phi^{(x, l)} e$ gives the probability for $l$ inventories in the system. The expected inventory level is given by

$$
\zeta_{I}=\sum_{x=0}^{\infty} \sum_{l=1}^{S} l \phi^{(x, l)} e .
$$

From the theorem 3.2 and the special structure of the $\boldsymbol{R}$ and $\boldsymbol{R}^{k}$ matrices, we simplify the above infinite summation by

$$
\zeta_{I}=\sum_{l=1}^{S} l\left[\phi^{(0, l)} e+\phi^{(1, l)} e+\phi^{(1,0)}\left[I-\boldsymbol{R}_{0}\right]^{-1} \boldsymbol{R}_{l} e\right] .
$$


2. Expected reorder rate Let $\zeta_{\boldsymbol{R}}$ denote the expected reorder rate in the steady state. We note that an order is placed when the inventory level drops to $s$. We also note that the inventory level drops to $s$ in the following cases:

(1) The inventory level is in $s+2$, and both events "the demand selection" and "the demand" take place.

(2) The inventory level is in $s+1$, and a demand takes place.

These lead to

$$
\zeta_{\boldsymbol{R}}=\sum_{x=0}^{\infty}\left(\phi^{(x, s+1)} D_{1}\right) e+\sum_{x=1}^{\infty}\left(\phi^{(x, s+2)}\left(D_{1} \otimes t_{2}\right)\right) e .
$$

The simplified version of $\zeta_{\boldsymbol{R}}$ is given by

$$
\begin{aligned}
\zeta_{\boldsymbol{R}}= & \phi^{(0, s+1)} D_{1} e+\phi^{(1, s+1)} D_{1} e+\phi^{(1, s+2)}\left(D_{1} \otimes t_{2}\right) e+\phi^{(1,0)}[I \\
& \left.-\boldsymbol{R}_{0}\right]^{-1}\left[\boldsymbol{R}_{s+1} D_{1} e+\boldsymbol{R}_{s+2}\left(D_{1} \otimes t_{2}\right) e\right] .
\end{aligned}
$$

3. Expected number of demands in the pool Let $\zeta_{P}$ denote the expected number of customers in the pool in the steady state. This is given by

$$
\zeta_{P}=\underline{\phi}^{(1)}(I-\boldsymbol{R})^{-2} e .
$$

4. Expected number of demands lost Let $\zeta_{L}$ denote the loss due to the customer who arrives during stock out and may not be willing to join the pool. Then $\zeta_{L}$ is given by

$$
\zeta_{L}=\sum_{x=0}^{\infty}\left(\phi^{(x, 0)}\left(q D_{1} \otimes T_{1}\right)\right) e .
$$

The $\zeta_{L}$ can be simplified as

$$
\zeta_{L}=\phi^{(0,0)}\left(q D_{1} \otimes T_{1}\right) e+\phi^{(1,0)}\left[I-\boldsymbol{R}_{0}\right]^{-1}\left(q D_{1} \otimes T_{1}\right) e .
$$

5. Expected total cost rate We assume the following costs in connection with maintaining the inventory.

$c_{\mathrm{h}}$ : holding cost of an item per unit time.

$c_{\mathrm{s}}$ : set-up cost per order.

$c_{\mathrm{W}}$ : waiting time cost of a demand per unit time in the pool.

$c_{l}$ : cost of loss of a demand due to stock out.

The expected total cost per unit time (the total expected cost rate) in the steady state for this model is defined to be

$$
T C(S, s)=c_{\mathrm{h}} \zeta_{I}+c_{\mathrm{s}} \zeta_{R}+c_{\mathrm{w}} \zeta_{P}+c_{l} \zeta_{L} .
$$

Substituting the values of '̧'s from Eqs. (4.1)-(4.4), we get 


$$
\begin{aligned}
& T C(S, s)= c_{\mathrm{h}}\left(\sum_{l=1}^{S} l\left[\phi^{(0, l)} e+\phi^{(1, l)} e+\phi^{(1,0)}\left[I-\boldsymbol{R}_{0}\right]^{-1} \boldsymbol{R}_{l} e\right]\right) \\
&+c_{\mathrm{s}}\left(\phi^{(0, s+1)} D_{1} e+\phi^{(1, s+1)} D_{1} e+\phi^{(1, s+2)}\left(D_{1} \otimes t_{2}\right) e+\phi^{(1,0)}\right. \\
& {\left.\left[I-\boldsymbol{R}_{0}\right]^{-1}\left[\boldsymbol{R}_{s+1} D_{1} e+\boldsymbol{R}_{s+2}\left(D_{1} \otimes t_{2}\right) e\right]\right)+c_{\mathrm{w}} \underline{\phi}^{(1)}(I-\boldsymbol{R})^{-2} e } \\
&+c_{1}\left(\phi^{(0,0)}\left(q D_{1} \otimes T_{1}\right) e+\phi^{(1,0)}\left[I-\boldsymbol{R}_{0}\right]^{-1}\left(q D_{1} \otimes T_{1}\right) e\right)
\end{aligned}
$$

Due to the complex form of the limiting distribution, it is difficult to discuss the qualitative behaviour of the cost function $\operatorname{TC}(S, s)$ analytically. Hence, a detailed computational study of the expected cost rate function is carried out in the next section.

\section{Numerical Illustrations}

In this section, we discuss some numerical examples that reveal the possible convexity of the total expected cost rate. For all the numerical works considered below, we assume that the arrival process is MAP specified by $\left(D_{0}, D_{1}\right)$ where

$$
D_{0}=\left[\begin{array}{ll}
0.2 & 0.0 \\
0.1 & 0.1
\end{array}\right], \quad D_{1}=\left[\begin{array}{ll}
0.7 & 0.1 \\
0.3 & 0.5
\end{array}\right] \text {. }
$$

The lead time has the phase-type distribution $\left(\alpha_{1}, T_{1}\right)$, where

$$
\alpha_{1}=[0.4,0.2,0.4], \quad T_{1}=\left[\begin{array}{ccc}
0.6 & 0.4 & 0 \\
0 & 0.6 & 0.4 \\
0 & 0 & 0.6
\end{array}\right] .
$$

The inter-selection times are assumed to have a phase-type distribution $\left(\alpha_{2}, T_{2}\right)$, where

$$
\alpha_{2}=[0.4,0.6], \quad T_{2}=\left[\begin{array}{ll}
0.3 & 0.7 \\
0.0 & 0.3
\end{array}\right] .
$$

For the model developed in this work, we have not shown the convexity of $T C(s, S)$ analytically. However, our experience with considerable numerical examples indicates that the function $T C(s, S)$ is convex. We use simple numerical search procedure to get the optimal values of total cost rate, $S$ and $s$ (say $T C^{*}, S^{*}, s^{*}$ ).

\section{Numerical Search Procedure:}

Step 1 By fixing $S$ and varying $s$, find that its corresponding total cost rates with the curve of total expected cost rate is to be convex.

Step 2 Do step 1 for different values of $S$. 
Table 3 Total expected cost rate as a function of $S$ and $s$

\begin{tabular}{|c|c|c|c|}
\hline \multirow{2}{*}{$\begin{array}{l}\mathrm{s} \\
\mathrm{S}\end{array}$} & \multicolumn{3}{|c|}{ Total expected cost rate } \\
\hline & 6 & 7 & 8 \\
\hline 22 & 1.9239733643 & $\underline{1.9057785631}$ & 1.9253718162 \\
\hline 23 & 1.9131864698 & $\underline{1.8951976818}$ & 1.9123311252 \\
\hline 24 & 1.9113560480 & $\underline{1.8941644154}$ & 1.9099202826 \\
\hline 25 & 1.9165365236 & $\underline{1.9004790337}$ & 1.9155371994 \\
\hline 26 & 1.9273133088 & 1.9125770010 & 1.9273764547 \\
\hline
\end{tabular}

$p=0.7, c_{\mathrm{h}}=0.1, c_{\mathrm{s}}=10, c_{w}=3, c_{l}=2$.

Step 3 Arrange the $T C$ values obtained in each step as the rows of a matrix, like Table 3.

Step 4 Find the minimum value for each row and each column.

Step 5 Find the minimum among the values obtained in step 4, the result gives the optimal total expected cost rate $T C^{*}$ and its corresponding $S$ and $s$ are the optimal $S^{*}$ and $s^{*}$.

In Table 3, the value that is shown bold is the least among the values in that column, and the value that is shown underlined is the least in that row. The minimum of bold and underlined value is the optimal total cost rate $T C^{*}$. The optimal cost value $T C^{*}=1: 8941644154$ is obtained at $\left(S^{*}, s^{*}\right)=(24,7)$ from Table 3 . A typical three-dimensional plot of the expected total cost function is shown in Fig. 1. It may be observed that these values in each table exhibit a (possibly) local minimum of the function of the two variables.

Next, we focus our attention to study the sensitivity to the costs $c_{\mathrm{h}}, c_{\mathrm{s}}, c_{\mathrm{w}}, c_{l}$ on the optimal values $S^{*}$ and $s^{*}$ and on the corresponding optimal cost, $T C^{*}$, in Tables 4 and 5. A summary of examination of the entries in this table is given below:

- We notice that the optimal cost appears to increase as $c_{\mathrm{h}}, c_{\mathrm{s}}, c_{\mathrm{w}}, c_{l}$ increase.

- As is to be expected, the optimal values of $S^{*}$ and $s^{*}$ decrease monotonically when the holding cost increases. This is because the holding cost increases, and we have to maintain low stock.

- We also notice that the optimal values of $S^{*}$ and $s^{*}$ increase monotonically when the cost of waiting in the pool and the cost of lost due to zero stock increase.

- It is interesting that as $c_{\mathrm{s}}$ increases, the optimum inventory level increases and the optimum reorder point decreases.

The observations made on Fig. 2 are listed below:

- The increment in the arrival rate decreases the mean inventory level of the system.

- As we increase the arrival rate of the customers, the expected number of reorder is increased. 


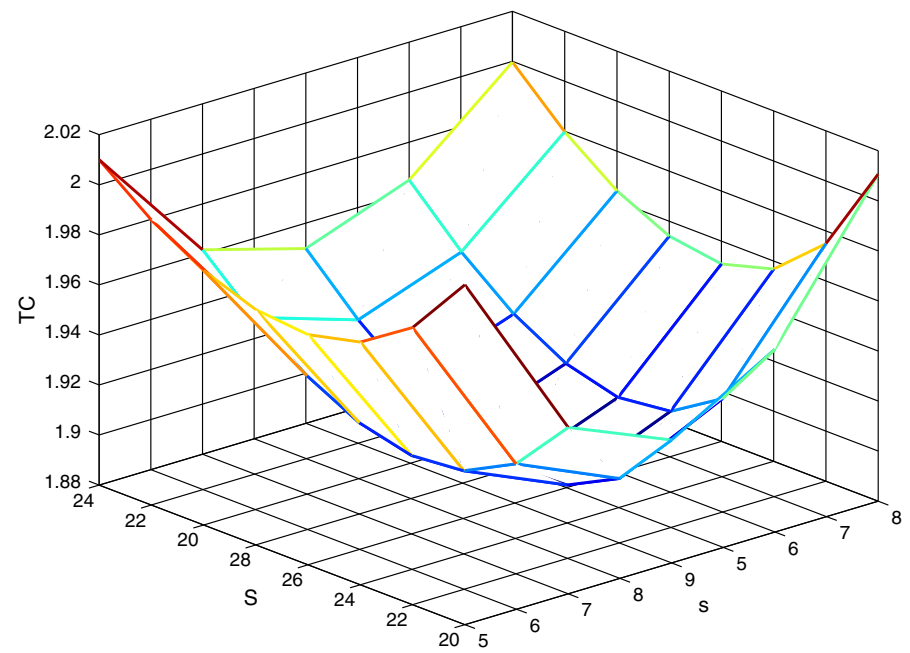

Fig. 1 A typical three-dimensional plot for convexity of total expected cost rate of $p=0.7, c_{\mathrm{h}}=0.1, c_{\mathrm{s}}=10, c_{\mathrm{w}}=3, c_{1}=2$

- The mean number of demands in the pool and the expected number of demands lost in the system increase when we increase the arrival rate of demands.

Finally, we study the impact of the value of probability $p$ on the total expected cost rate through the Figs. 3 and 4. We notice that as the $p$ value increases, the total cost increases. For the small values of $p$ means, the number of customers who accept the offer of postponed their demand is less, and hence they approach another place for their demand. In order to avoid this situation, we have to maintain large inventory. This will be reflected in Fig. 3. Similar reason holds good for Fig. 4 also.

\section{Conclusion}

In the literature, the Inventory systems have been monitored either periodically or continuously. In the former, the duration of a period could be a day, a week or a month and in the latter, every transaction that induces some changes in the system is assumed to be available. However, in recent time due to advances in transaction reporting devices, the time axis is slotted with smaller durations, and any incidents such as demand, etc., which occur in these slots, are synchronized to have occurred at any one of the end points of the slot. The telecommunication and computer networks, which are becoming digital, have initiated interest in the systems which are monitored in slotted time axis.

In this work, we modelled an inventory system in a slotted time axis with an infinite pool. The arrival time points of demands assumed to be generated by a DMAP which actually provided a basic setup for correlated demand types, and thereby the model provided to be used to include some non-Markovian demand 
Table 4 Effect of costs on the optimal values

\begin{tabular}{|c|c|c|c|c|c|c|c|c|c|c|c|c|c|c|c|}
\hline \multirow{4}{*}{$c_{w}$} & \multirow{4}{*}{$\mathrm{c}_{1}$} & \multicolumn{14}{|l|}{$c_{h}$} \\
\hline & & \multicolumn{8}{|l|}{0.1} & \multicolumn{6}{|l|}{0.2} \\
\hline & & \multicolumn{14}{|l|}{$\mathrm{c}_{\mathrm{s}}$} \\
\hline & & 8 & & 10 & & 12 & & 14 & & 8 & 10 & & 12 & 14 & \\
\hline \multirow[t]{8}{*}{2.5} & 1.5 & 22 & 7 & 23 & 7 & 24 & 6 & 25 & 6 & 19 & 19 & 6 & 20 & 20 & 5 \\
\hline & & 1.7539 & & 1.8542 & 2 & 1.9472 & 2 & 2.0352 & 2 & 2.7419 & 2.864 & & 2.9741 & 3.079 & \\
\hline & 2 & 227 & 7 & 23 & 7 & 24 & 6 & 256 & 6 & 196 & 19 & 6 & $20 \quad 5$ & 20 & 5 \\
\hline & & 1.7559 & & 1.856 & & 1.9497 & 7 & 2.0375 & & 2.7454 & 2.867 & 4 & 2.9787 & 3.0841 & \\
\hline & 2.5 & 227 & 7 & 237 & 7 & 246 & 6 & 25 & 6 & 196 & 19 & 6 & 20 & 20 & 5 \\
\hline & & 1.7579 & & 1.8579 & & 1.9522 & 2 & 2.0399 & & 2.7489 & 2.870 & 9 & 2.9832 & 3.088 & \\
\hline & 3 & 227 & 7 & 23 & 7 & 24 & 6 & 25 & 6 & 19 & 19 & 6 & $20 \quad 5$ & 20 & 5 \\
\hline & & 1.7598 & & 1.8597 & 7 & 1.9548 & & 2.0423 & & 2.7524 & 2.874 & 4 & 2.9877 & 3.0932 & 2 \\
\hline \multirow[t]{8}{*}{3} & 1.5 & 23 & 7 & 24 & 7 & 24 & 7 & 25 & 7 & 20 & 20 & 6 & 20 & 21 & 6 \\
\hline & & 1.7938 & & 1.8924 & & 1.9861 & 1 & 2.0758 & & 2.8269 & 2.940 & 3 & 3.0537 & 3.1645 & \\
\hline & 2 & 237 & 7 & 247 & 7 & 24 & 7 & 257 & 7 & $20 \quad 6$ & 20 & 6 & 20 & 21 & 6 \\
\hline & & 1.7956 & & 1.8941 & 1 & 1.9878 & & 2.0775 & & 2.8302 & 2.943 & 5 & 3.0569 & 3.1675 & \\
\hline & 2.5 & 23 & 7 & 24 & 7 & 24 & 7 & 25 & 7 & 20 & 20 & 6 & 20 & 21 & \\
\hline & & 1.7975 & & 1.8959 & 9 & 1.9896 & & 2.0791 & & 2.8334 & 2.946 & & 3.0601 & 3.170 & \\
\hline & 3 & 23 & 7 & 24 & 7 & 24 & 7 & 25 & 7 & 20 & 20 & 6 & 206 & 21 & 6 \\
\hline & & 1.7993 & & 1.8976 & & 1.9913 & & 2.0808 & & 2.8366 & 2.95 & & 3.0634 & 3.173 & \\
\hline \multirow[t]{8}{*}{3.5} & 1.5 & 23 & 7 & 24 & 7 & 25 & 7 & 25 & 7 & 206 & 20 & 6 & 216 & 21 & \\
\hline & & 1.8329 & & 1.9279 & & 2.0199 & & 2.1084 & & 2.9013 & 3.014 & & 3.1246 & 3.230 & \\
\hline & 2 & 23 & 7 & 24 & 7 & 25 & 7 & 25 & 7 & 20 & 20 & 6 & 21 & 21 & \\
\hline & & 1.8347 & & 1.9296 & 6 & 2.0215 & 5 & 2.11 & & 2.9045 & 3.017 & 9 & 3.1276 & 3.233 & \\
\hline & 2.5 & 23 & 8 & 24 & 7 & 25 & 7 & 25 & 7 & 20 & 20 & 6 & 216 & 21 & \\
\hline & & 1.8361 & 1 & 1.9314 & 4 & 2.0232 & 2 & 2.1117 & 7 & 2.9077 & 3.021 & 1 & 3.1306 & 3.236 & \\
\hline & 3 & 23 & 8 & 24 & 7 & 25 & 7 & 25 & 7 & 20 & 20 & 6 & 216 & 21 & \\
\hline & & 1.8374 & & 1.9331 & 1 & 2.0248 & & 2.1133 & & 2.911 & 3.024 & 3 & 3.1337 & 3.239 & \\
\hline \multirow[t]{8}{*}{4} & 1.5 & 24 & 8 & 24 & 8 & 25 & 7 & 26 & 7 & 20 & 21 & 7 & 21 & 21 & \\
\hline & & 1.8603 & 3 & 1.96 & & 2.0524 & 4 & 2.139 & & 2.9641 & 3.080 & 6 & 3.1906 & 3.296 & \\
\hline & 2 & 24 & 6 & 24 & 8 & 25 & 7 & 26 & 7 & 20 & 21 & 7 & 21 & 21 & \\
\hline & & 1.8615 & 5 & 1.9612 & 2 & 2.0541 & 1 & 2.1406 & & 2.9664 & 3.082 & & 3.1936 & 3.299 & \\
\hline & 2.5 & 24 & 8 & 24 & 8 & 25 & 7 & 26 & 7 & $20 \quad 7$ & 21 & 7 & 216 & 21 & \\
\hline & & 1.8627 & & 1.9624 & & 2.0557 & & 2.1422 & & 2.9686 & 3.084 & & 3.1966 & 3.3025 & \\
\hline & 3 & 24 & 8 & 24 & 8 & 25 & 7 & 26 & 7 & $20 \quad 7$ & 21 & 7 & 21 & 21 & \\
\hline & & 1.8639 & & 1.9636 & & 2.0574 & & 2.1437 & & 2.9709 & 3.087 & & 3.1996 & 3.305 & \\
\hline
\end{tabular}


Table 5 Effect of costs on the optimal values

\begin{tabular}{|c|c|c|c|c|c|c|c|c|c|c|c|c|c|c|c|c|}
\hline \multirow{4}{*}{$\mathrm{c}_{\mathrm{w}}$} & \multirow{4}{*}{$\mathrm{c}_{1}$} & \multicolumn{15}{|l|}{$c_{h}$} \\
\hline & & \multicolumn{8}{|l|}{0.3} & \multicolumn{7}{|l|}{0.4} \\
\hline & & \multicolumn{15}{|l|}{$\mathrm{c}_{\mathrm{s}}$} \\
\hline & & \multicolumn{2}{|l|}{8} & \multicolumn{2}{|l|}{10} & \multicolumn{2}{|l|}{12} & \multicolumn{2}{|l|}{14} & \multicolumn{2}{|l|}{8} & 10 & \multicolumn{2}{|l|}{12} & \multicolumn{2}{|l|}{14} \\
\hline \multirow[t]{8}{*}{2.5} & 1.5 & 17 & 5 & 18 & 5 & 18 & 5 & 18 & 5 & 16 & 5 & 16 & 17 & 4 & 17 & 4 \\
\hline & & 3.576 & 2 & 3.702 & & 3.824 & & 3.9455 & 5 & 4.3359 & 9 & 4.4681 & 4.589 & 1 & 4.709 & 8 \\
\hline & 2 & 17 & 5 & 18 & 5 & 18 & 5 & 18 & 5 & 16 & 5 & 17 & 17 & 4 & 17 & 4 \\
\hline & & 3.581 & 8 & 3.707 & 8 & 3.8292 & 2 & 3.9507 & & 4.342 & & 4.476 & 4.596 & 7 & 4.717 & 5 \\
\hline & 2.5 & 17 & 5 & 18 & 5 & 18 & 5 & 18 & 5 & 16 & 5 & 17 & 17 & 4 & 17 & 4 \\
\hline & & 3.587 & 5 & 3.713 & & 3.8345 & 5 & 3.9559 & & 4.3482 & 2 & 4.4836 & 4.604 & 4 & 4.725 & 1 \\
\hline & 3 & 17 & 5 & 18 & 5 & 18 & 5 & 18 & 5 & 16 & 5 & 17 & 17 & 4 & 17 & 4 \\
\hline & & 3.593 & 1 & 3.718 & 2 & 3.8397 & 7 & 3.9612 & 2 & 4.3543 & 3 & 4.4893 & 4.612 & 1 & 4.732 & 8 \\
\hline \multirow[t]{8}{*}{3} & 1.5 & 18 & 5 & 18 & 5 & 18 & 5 & 19 & 5 & 17 & 5 & 17 & 17 & 5 & 17 & 4 \\
\hline & & 3.707 & 7 & 3.829 & 1 & 3.9506 & 6 & 4.0687 & 7 & 4.4888 & 8 & 4.6202 & 4.751 & 7 & 4.883 & 1 \\
\hline & 2 & 18 & 5 & 18 & 5 & 18 & 5 & 19 & 5 & 17 & 5 & 17 & 17 & 5 & 17 & 4 \\
\hline & & 3.712 & 9 & 3.834 & 3 & 3.9558 & & 4.0736 & & 4.4944 & 4 & 4.6259 & 4.757 & 3 & 4.888 & \\
\hline & 2.5 & 18 & 5 & 18 & 5 & 18 & 5 & 19 & 5 & 17 & 5 & 17 & 17 & 5 & 17 & 5 \\
\hline & & 3.718 & 1 & 3.839 & 6 & 3.961 & & 4.0784 & 4 & 4.5001 & 1 & 4.6315 & 4.763 & & 4.894 & \\
\hline & 3 & 18 & 5 & 18 & 5 & 18 & 5 & 19 & 5 & 17 & 5 & 17 & 17 & 5 & 17 & 5 \\
\hline & & 3.723 & 3 & 3.844 & & 3.9663 & & 4.0833 & & 4.5057 & 7 & 4.6372 & 4.768 & 6 & 4.900 & 1 \\
\hline \multirow[t]{8}{*}{3.5} & 1.5 & 18 & 6 & 19 & 6 & 19 & 6 & 19 & 6 & 17 & 5 & 17 & 18 & 5 & 18 & 5 \\
\hline & & 3.815 & 5 & 3.943 & & 4.065 & & 4.187 & & 4.6366 & & 4.7681 & 4.892 & 7 & 5.014 & \\
\hline & 2 & 18 & 6 & 19 & 6 & 19 & 6 & 19 & 6 & 17 & 5 & 17 & 18 & 5 & 18 & 5 \\
\hline & & 3.819 & 2 & 3.946 & 5 & 4.0685 & 5 & 4.1905 & 5 & 4.6423 & 3 & 4.7737 & 4.898 & & 5.019 & 4 \\
\hline & 2.5 & 18 & 6 & 19 & 6 & 19 & 6 & 19 & 6 & 17 & 5 & 17 & 18 & 5 & 18 & 5 \\
\hline & & 3.823 & & 3.95 & & 4.072 & & 4.194 & & 4.6479 & 9 & 4.7794 & 4.903 & 2 & 5.024 & 7 \\
\hline & 3 & 18 & 6 & 19 & 6 & 19 & 6 & 19 & 6 & 17 & 5 & 17 & 18 & 5 & 18 & 5 \\
\hline & & 3.826 & 8 & 3.953 & 4 & 4.0755 & & 4.1975 & & 4.6536 & 6 & 4.785 & 4.908 & 4 & 5.029 & \\
\hline \multirow[t]{8}{*}{4} & 1.5 & 19 & 6 & 19 & 6 & 19 & 6 & 19 & 6 & 18 & 6 & 18 & 18 & 5 & 18 & 5 \\
\hline & & 3.906 & 4 & 4.028 & 4 & 4.1504 & 4 & 4.2725 & & 4.7733 & 3 & 4.8978 & 5.019 & 3 & 5.140 & \\
\hline & 2 & 19 & 6 & 19 & 6 & 19 & 6 & 19 & 6 & 18 & 6 & 18 & 18 & 5 & 18 & 5 \\
\hline & & 3.909 & 9 & 4.031 & 9 & 4.1539 & 9 & 4.2759 & & 4.7771 & 1 & 4.9031 & 5.024 & 5 & 5.146 & \\
\hline & 2.5 & 19 & 6 & 19 & 6 & 19 & 6 & 19 & 6 & 18 & 6 & 18 & 18 & 5 & 18 & 5 \\
\hline & & 3.913 & 4 & 4.035 & 4 & 4.1574 & 4 & 4.2794 & 4 & 4.7808 & 8 & 4.9083 & 5.029 & 8 & 5.151 & 2 \\
\hline & 3 & 19 & 6 & 19 & 6 & 19 & 6 & 19 & 6 & 18 & 6 & 18 & 18 & 5 & 18 & 5 \\
\hline & & 3.916 & & 4.038 & & 4.1609 & & 4.2829 & & 4.7846 & & 4.9135 & 5.035 & & 5.156 & \\
\hline
\end{tabular}



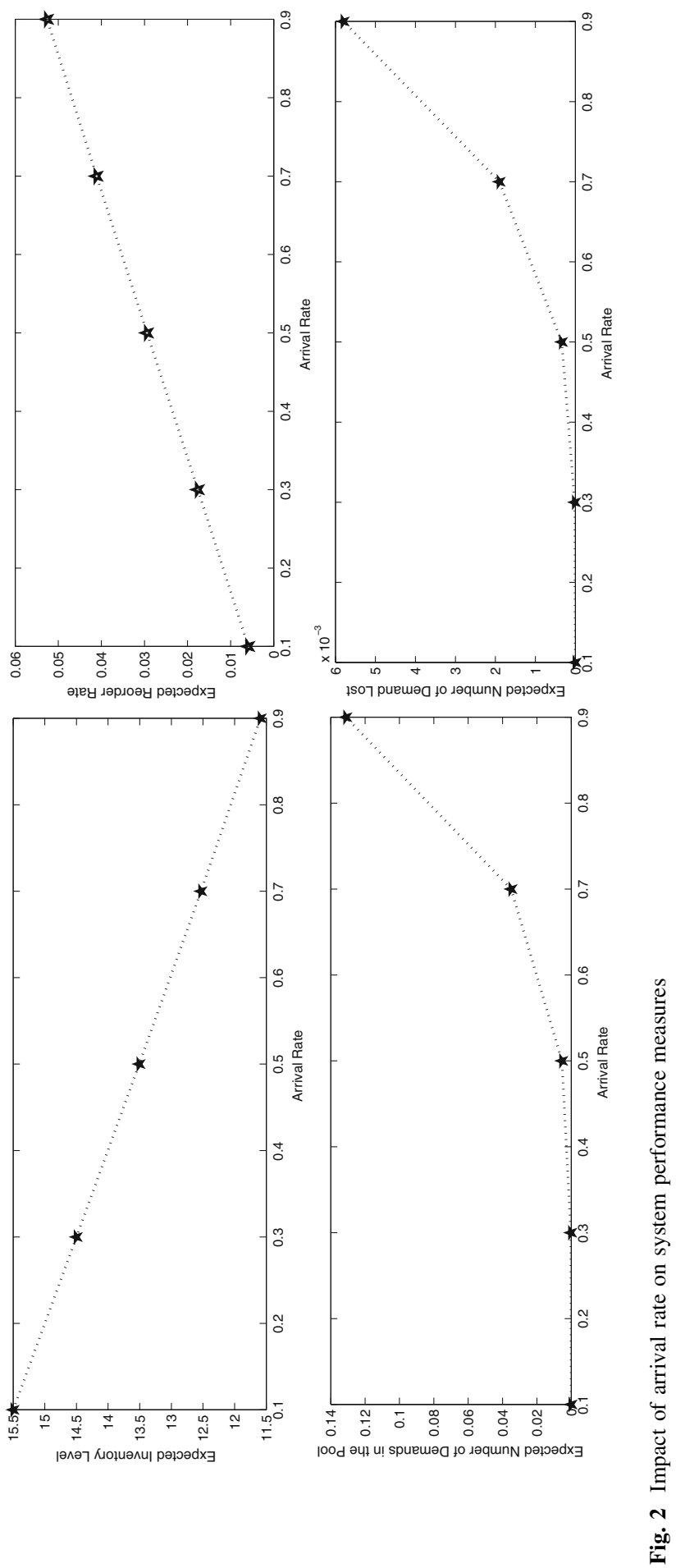


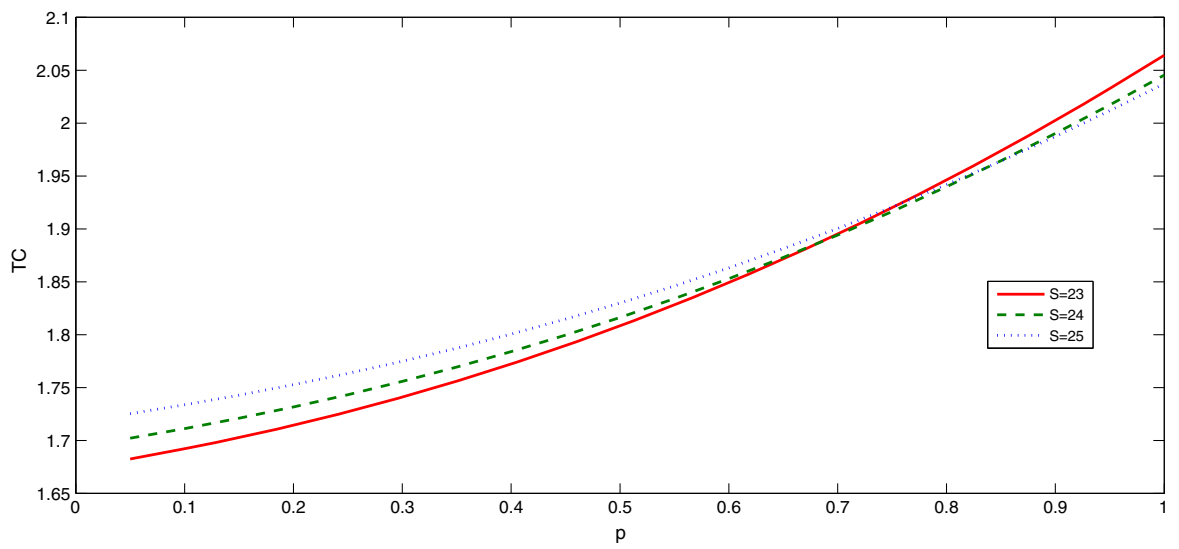

Fig. 3 Total expected cost rate as a function of $p s=7, c_{\mathrm{h}}=0.1, c_{\mathrm{s}}=10, c_{\mathrm{w}}=3, c_{1}=2$

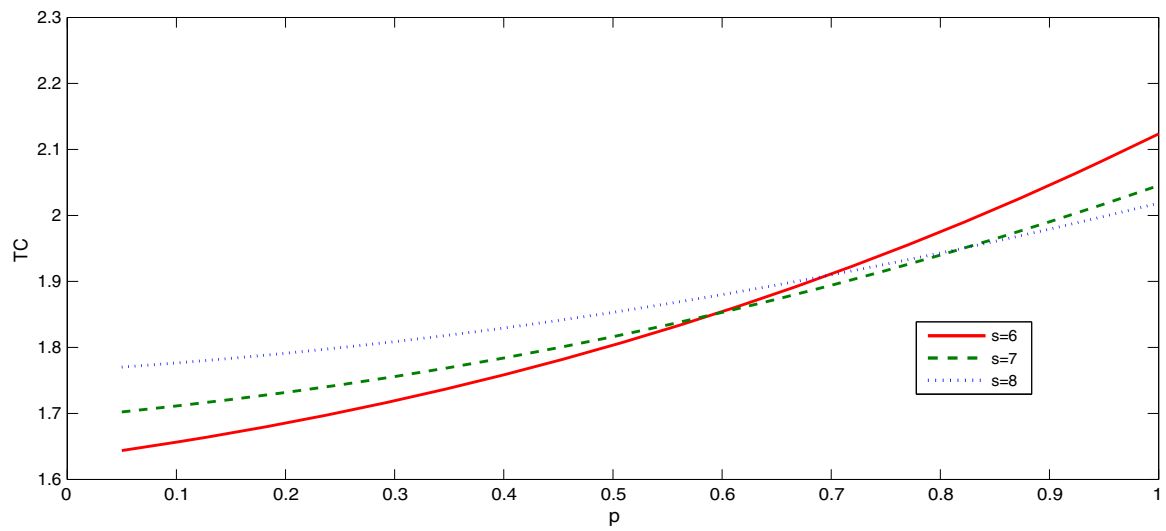

Fig. 4 Total expected cost rate as a function of p $S=24, c_{\mathrm{h}}=0.1, c_{\mathrm{s}}=10, c_{\mathrm{w}}=3, c_{1}=2$

processes. The assumptions of phase-type distributions for lead time and for interselection time for demands allowed us to fit them to a wide range of real-life situations. We obtained the limiting probability of the inventory level in the pool. Using this, we derived some of the system performances. Also, we calculated expected total cost rate of the system under a suitable cost structure. The convex nature of the expected total cost rate is shown numerically.

Acknowledgments The authors wish to thank the anonymous referees who have provided valuable suggestions for improving the presentation of this article, and also we thank the associate editor Qi-Ming He for his valuable suggestions to improve the quality of the article. 


\section{Appendix 1}

To compute the $\boldsymbol{R}$ matrix, we use the following set of non-linear equations. This can be solved using the Gauss-Seidel iterative process. These equations are derived by exploiting the coefficient matrices appearing in (3.3).

For $i=0$,

$$
\boldsymbol{R}_{(i)}\left(D_{0} \otimes T_{1}+q D_{1} \otimes T_{1}\right)+\boldsymbol{R}_{(i+1)}\left(D_{1} \otimes T_{1}\right)+p D_{1} \otimes T_{1}=\boldsymbol{R}_{(0)} .
$$

For $i=1,2, \cdots, s-1$,

$$
\boldsymbol{R}_{(i)}\left(D_{0} \otimes T_{1}\right)+\boldsymbol{R}_{(i+1)}\left(D_{1} \otimes T_{1}\right)=\boldsymbol{R}_{(i)} .
$$

For $i=s$,

$$
\boldsymbol{R}_{(i)}\left(D_{0} \otimes T_{1}\right)+\boldsymbol{R}_{(i+1)}\left(D_{1} \otimes \alpha_{1}\right)+\boldsymbol{R}_{(i+2)}\left(D_{1} \otimes \alpha_{1} \otimes t_{2}\right)=\boldsymbol{R}_{(i)} .
$$

For $i=s+1$,

$$
\boldsymbol{R}_{(i)} D_{0}+\boldsymbol{R}_{(i+1)}\left(D_{1} \otimes T_{2} e_{m_{2}}\right)+\boldsymbol{R}_{(0)} \boldsymbol{R}_{(i+1)}\left(D_{0} \otimes t_{2}\right)+\boldsymbol{R}_{(0)} \boldsymbol{R}_{(i+2)}\left(D_{1} \otimes t_{2}\right)=\boldsymbol{R}_{(i)} .
$$

For $i=s+2, s+3 \cdots, Q-2$,

$$
\begin{aligned}
& \boldsymbol{R}_{(i)}\left(D_{0} \otimes T_{2}\right)+\boldsymbol{R}_{(i+1)}\left(D_{1} \otimes T_{2}\right)+\boldsymbol{R}_{(0)} \boldsymbol{R}_{(i+1)}\left(D_{0} \otimes t_{2} \alpha_{2}\right)+\boldsymbol{R}_{(0)} \boldsymbol{R}_{(i+2)}\left(D_{1} \otimes t_{2} \alpha_{2}\right) \\
& \quad=\boldsymbol{R}_{(i)} .
\end{aligned}
$$

For $i=Q-1$,

$$
\begin{aligned}
& \boldsymbol{R}_{(0)}\left(D_{1} \otimes t_{1} \otimes \alpha_{2}\right)+\boldsymbol{R}_{(i)}\left(D_{0} \otimes T_{2}\right)+\boldsymbol{R}_{(i+1)}\left(D_{1} \otimes T_{2}\right)+\boldsymbol{R}_{(0)} \boldsymbol{R}_{(i+1)}\left(D_{0} \otimes t_{2} \alpha_{2}\right) \\
& \quad+\boldsymbol{R}_{(0)} \boldsymbol{R}_{(i+2)}\left(D_{1} \otimes t_{2} \alpha_{2}\right)=\boldsymbol{R}_{(i)} .
\end{aligned}
$$

For $i=Q, Q+1 \cdots, S-2$,

$$
\begin{aligned}
& \boldsymbol{R}_{(i-Q)}\left(D_{0} \otimes t_{1} \otimes \alpha_{2}\right)+\boldsymbol{R}_{(i-Q+1)}\left(D_{1} \otimes t_{1} \otimes \alpha_{2}\right)+\boldsymbol{R}_{(i)}\left(D_{0} \otimes T_{2}\right) \\
& \quad+\boldsymbol{R}_{(i+1)}\left(D_{1} \otimes T_{2}\right)+\boldsymbol{R}_{(0)} \boldsymbol{R}_{(i+1)}\left(D_{0} \otimes t_{2} \alpha_{2}\right)+\boldsymbol{R}_{(0)} \boldsymbol{R}_{(i+2)}\left(D_{1} \otimes t_{2} \alpha_{2}\right)=\boldsymbol{R}_{(i)} .
\end{aligned}
$$

For $i=S-1$,

$$
\begin{gathered}
\boldsymbol{R}_{(i-Q)}\left(D_{0} \otimes t_{1} \otimes \alpha_{2}\right)+\boldsymbol{R}_{(i-Q+1)}\left(D_{1} \otimes t_{1} \alpha_{2}\right)+\boldsymbol{R}_{(i)}\left(D_{0} \otimes T_{2}\right) \\
\quad+\boldsymbol{R}_{(i+1)}\left(D_{1} \otimes T_{2}\right)+\boldsymbol{R}_{(0)} \boldsymbol{R}_{(i+1)}\left(D_{0} \otimes t_{2} \alpha_{2}\right)=\boldsymbol{R}_{(i)} .
\end{gathered}
$$

For $i=S$,

$$
\boldsymbol{R}_{(i-Q)}\left(D_{0} \otimes t_{1} \otimes \alpha_{2}\right)+\boldsymbol{R}_{(i)}\left(D_{0} \otimes T_{2}\right)=\boldsymbol{R}_{(i)} .
$$




\section{Appendix 2}

To compute the $\underline{\phi}^{(0)}$ and $\underline{\phi}^{(1)}$ matrices, we use the following set of non-linear equations. From Eq. (3.4), we get

For $i=0$,

$$
\phi^{(0, i)}\left(D_{0} \otimes T_{1}+q D_{1} \otimes T_{1}\right)+\phi^{(0, i+1)}\left(D_{1} \otimes T_{1}\right)=\phi^{(0, i)} .
$$

For $i=1,2, \cdots, s-1$,

$$
\phi^{(0, i)}\left(D_{0} \otimes T_{1}\right)+\phi^{(0, i+1)}\left(D_{1} \otimes T_{1}\right)=\phi^{(0, i)} .
$$

For $i=s$,

$$
\phi^{(0, i)}\left(D_{0} \otimes T_{1}\right)+\phi^{(0, i+1)}\left(D_{1} \otimes \alpha_{1}\right)+\phi^{(1, i+2)}\left(D_{1} \otimes \alpha_{1} \otimes t_{2}\right)=\phi^{(0, i)} .
$$

For $i=s+1, s+2, \cdots, Q-2$,

$$
\phi^{(0, i)} D_{0}+\phi^{(0, i+1)} D_{1}+\phi^{(1, i+1)}\left(D_{0} \otimes t_{2}\right)+\phi^{(1, i+2)}\left(D_{1} \otimes t_{2}\right)=\phi^{(0, i)} .
$$

For $i=Q-1$,

$$
\phi^{(0,0)}\left(D_{1} \otimes t_{1}\right)+\phi^{(0, i)} D_{0}+\phi^{(0, i+1)} D_{1}+\phi^{(1, i+1)}\left(D_{0} \otimes t_{2}\right)+\phi^{(1, i+2)}\left(D_{1} \otimes t_{2}\right)=\phi^{(0, i)} .
$$

For $i=Q, Q+1, \cdots, S-2$,

$$
\begin{aligned}
& \phi^{(0, i-Q)}\left(D_{0} \otimes t_{1}\right)+\phi^{(0, i-Q+1)}\left(D_{1} \otimes t_{1}\right)+\phi^{(0, i)} D_{0}+\phi^{(0, i+1)} D_{1} \\
& \quad+\phi^{(1, i+1)}\left(D_{0} \otimes t_{2}\right)+\phi^{(1, i+2)}\left(D_{1} \otimes t_{2}\right)=\phi^{(0, i)} .
\end{aligned}
$$

For $i=S-1$,

$$
\begin{aligned}
& \phi^{(0, i-Q)}\left(D_{0} \otimes t_{1}\right)+\phi^{(0, i-Q+1)}\left(D_{1} \otimes t_{1}\right)+\phi^{(0, i)} D_{0}+\phi^{(0, i+1)} D_{1} \\
& \quad+\phi^{(1, i+1)}\left(D_{0} \otimes t_{2}\right)=\phi^{(0, i)} .
\end{aligned}
$$

For $i=S$,

$$
p h i^{(0, i-Q)}\left(D_{0} \otimes t_{1}\right)+\phi^{(0, i)} D_{0}=\phi^{(0, i)} .
$$

From Eq. (3.5) we get, For $i=0$,

$$
\phi^{(1, i)}\left(D_{0} \otimes T_{1}+q D_{1} \otimes T_{1}\right)+\phi^{(1, i+1)}\left(D_{1} \otimes T_{1}\right)+\phi^{(0, i)}\left(p D_{1} \otimes T_{1}\right)=\phi^{(1, i)} .
$$

For $i=1,2, \cdots, s-1$,

$$
\phi^{(1, i)}\left(D_{0} \otimes T_{1}\right)+\phi^{(1, i+1)}\left(D_{1} \otimes T_{1}\right)=\phi^{(1, i)} .
$$


For $i=s$,

$$
\phi^{(1, i)}\left(D_{0} \otimes T_{1}\right)+\phi^{(1, i+1)}\left(D_{1} \otimes \alpha_{1}\right)+\phi^{(1,0)} \boldsymbol{R}_{(i+2)}\left(D_{1} \otimes \alpha_{1} \otimes t_{2}\right)=\phi^{(1, i)} .
$$

For $i=s+1$,

$$
\phi^{(1, i)} D_{0}+\phi^{(1, i+1)}\left(D_{1} \otimes T_{2} e_{m_{2}}\right)+\phi^{(1,0)} \boldsymbol{R}_{(i+1)}\left(D_{0} \otimes t_{2}\right)+\phi^{(1,0)} \boldsymbol{R}_{(i+2)}\left(D_{1} \otimes t_{2}\right)=\phi^{(1, i)} .
$$

For $i=s+2, s+3, \cdots, Q-2$,

$$
\begin{aligned}
& \phi^{(1, i)}\left(D_{0} \otimes T_{2}\right)+\phi^{(1, i+1)}\left(D_{1} \otimes T_{2}\right)+\phi^{(1,0)} \boldsymbol{R}_{(i+1)}\left(D_{0} \otimes t_{2} \alpha_{2}\right) \\
& +\phi^{(1,0)} \boldsymbol{R}_{(i+2)}\left(D_{1} \otimes t_{2} \alpha_{2}\right)=\phi^{(1, i)} .
\end{aligned}
$$

For $i=Q-1$,

$$
\begin{aligned}
& \phi^{(1,0)}\left(D_{1} \otimes t_{1} \otimes \alpha_{2}\right)+\phi^{(1, i)}\left(D_{0} \otimes T_{2}\right)+\phi^{(1, i+1)}\left(D_{1} \otimes T_{2}\right) \\
& \quad+\phi^{(1,0)} \boldsymbol{R}_{(i+1)}\left(D_{0} \otimes t_{2} \alpha_{2}\right)+\phi^{(1,0)} \boldsymbol{R}_{(i+2)}\left(D_{1} \otimes t_{2} \alpha_{2}\right)=\phi^{(1, i)} .
\end{aligned}
$$

For $i=Q, Q+1, \cdots, S-2$,

$$
\begin{aligned}
& \phi^{(1, i-Q)}\left(D_{0} \otimes t_{1} \otimes \alpha_{2}\right)+\phi^{(1, i-Q+1)}\left(D_{1} \otimes t_{1} \otimes \alpha_{2}\right)+\phi^{(1, i)}\left(D_{0} \otimes T_{2}\right) \\
& \quad+\phi^{(1, i+1)}\left(D_{1} \otimes T_{2}\right) \\
& \quad+\phi^{(1,0)} \boldsymbol{R}_{(i+1)}\left(D_{0} \otimes t_{2} \alpha_{2}\right)+\phi^{(1,0)} \boldsymbol{R}_{(i+2)}\left(D_{1} \otimes t_{2} \alpha_{2}\right)=\phi^{(1, i)} .
\end{aligned}
$$

For $i=S-1$,

$$
\begin{aligned}
& \phi^{(1, i-Q)}\left(D_{0} \otimes t_{1} \otimes \alpha_{2}\right)+\phi^{(1, i-Q+1)}\left(D_{1} \otimes t_{1} \otimes \alpha_{2}\right)+\phi^{(1, i)}\left(D_{0} \otimes T_{2}\right) \\
& +\phi^{(1, i+1)}\left(D_{1} \otimes T_{2}\right)+\phi^{(1,0)} \boldsymbol{R}_{(i+1)}\left(D_{0} \otimes t_{2} \alpha_{2}\right)=\phi^{(1, i)} .
\end{aligned}
$$

For $i=S$,

$$
\phi^{(1, i-Q)}\left(D_{0} \otimes t_{1} \otimes \alpha_{2}\right)+\phi^{(1, i)}\left(D_{0} \otimes T_{2}\right)=\phi^{(1, i)} .
$$

\section{References}

[1] Abboud, N.E.: A discrete-time markov production-inventory model with machine breakdowns. Comput. Ind. Eng. 39, 95-107 (2001)

[2] Alfa, A.S.: Discrete time queues and matrix-analytic methods. Top 10(2), 147-185 (2002)

[3] Alfa, A.S.: Vacation models in discrete time. Queueing Syst. 44, 5-30 (2003)

[4] Artalejo, J.R., Economou, A., Gómez-Corral, A.: Algorithmic analysis of the geo/geo/c retrial queue. Eur. J. Oper. Res. 189(3), 1042-1056 (2008)

[5] Asmussen, S.: Phase-type representations in random walk and queueing problems. Ann. Probab. 20, 772-789 (1992)

[6] Asmussen, S.: Ruin Probabilities. World Scientific, Singapore (2000)

[7] Bar-Lev, S.K., Perry, D.A.: Discrete time Markovian inventory model for perishable commodities. Stoch. Anal. Appl. 7, 243-259 (1989)

[8] Chakravarthy, S.R.: The batch Markovian arrival process: a review and future work. In: Krishnamoorthy, A., et al. (eds.) Advances in Probability and Stochastic Processes, pp. 21-49. Notable Publications, New Jersey (2001) 
[9] Dafermos, S.C., Neuts, M.F.: A single server queue in discrete time. Cah. Cent. Rech. Oper. 13, 23-40 (1971)

[10] Eisele, K.T.: Recursions for compound phase distributions. Insur. Math. Econ. 38, 149-156 (2006)

[11] Hunter, J.J.: Mathematical Techniques of Applied Probability, Vol. 2, Discrete Time Models: Techniques and Applications. Operations Research and Industrial Engineering Series Academic Press, New York (1983).

[12] Krishnamoorthy, A., Islam, M.E.: $(s, s)$ inventory system with postponed demands. Stoch. Anal. Appl. 22(3), 827-842 (2004)

[13] Latouche, G., Ramaswami, V.: Introduction to matrix analytic methods in stochastic modeling. ASA SIAM, Philadelphia (1999)

[14] Lian, Z., Liu, L.: A discrete-time model for perishable inventory systems. Ann. Oper. Res. 87, 103-116 (1999)

[15] Lian, Z., Liu, L., Neuts, M.F.: A discrete-time model for common lifetime inventory systems. Math.Oper. Res. 30(3), 718-732 (2005)

[16] Lucantoni, D.M.: New results on the single server queue with a batch Markovian arrival process. Stoch. Models 7, 1-46 (1991)

[17] Lucantoni, D.M., Meir-Hellstern, K.S., Neuts, M.F.: A single server queue with server vacations and a class of non-renewal arrival processes. Adv. Appl. Probab. 22, 676-705 (1990)

[18] Manuel, P., Sivakumar, B., Arivarignan, G.: Perishable inventory system with postponed demands and negative customers. J. Appl. Math. Decis. Sci. 2007, 1-12 (2007)

[19] Neuts, M.F.: Probability Distributions of Phase Type. In: Liber Amicorum, pp. 173-206. Emeritus H. Florin. Department of Mathematics University of Louvain, Belgium (1975)

[20] Neuts, M.F.: A versatile Markovian point process. J. Appl. Probab. 16, 764-779 (1979)

[21] Neuts, M.F.: Matrix-Geometric Solutions in Stochastic Models: An Algorithmic Approach. Johns Hopkins University Press, Baltimore (1981)

[22] Neuts, M.F.: Models based on the Markovian arrival process. IEICE Trans. Commun. E75-8, 1253-1265 (1992)

[23] Sivakumar, B., Arivarignan, G.: An inventory system with postponed demands. Stoch. Anal. Appl. 22(3), 827-842 (2008)

[24] Sivakumar, B., Arivarignan, G.: A stochastic inventory system with postponed demands. Perform. Eval. 66, 47-58 (2009)

[25] Sivakumar, B., Jayaraman, R., Arivarignan, G.: A discrete time inventory system with postponed demands. J. Comput. Appl. Math. 236, 3073-3083 (2012)

[26] Takagi, H.: Queueing Analysis-A foundation of Performance Evaluation. Elsevier Science Publishers B.V., Amsterdam (1993) 